\title{
Alteration of laboratory findings after radiofrequency ablation of hepatocellular carcinoma: relationship to severity of the underlying liver disease and the ablation volume
}

\author{
Sang-Wook Shin', Woo Kyoung Jeong ${ }^{1,2}$, Sanghyeok Lim', Yongsoo Kim', and Jinoo Kim ${ }^{1,3}$ \\ 'Department of Radiology, Hanyang University Guri Hospital, Hanyang University College of Medicine, Guri; ${ }^{2}$ Department of Radiology \\ and Center for Imaging Science, Samsung Medical Center, Sungkyunkwan University, School of Medicine, Seoul; ${ }^{3}$ Department of \\ Radiology, Ajou University Hospital, Ajou University College of Medicine, Suwon, Korea
}

Background/Aims: To investigate sequential changes in laboratory markers after radiofrequency ablation (RFA) of hepatocellular carcinoma (HCC) and the relationship of these changes to the severity of the underlying liver disease.

Methods: This retrospective analysis included 65 patients (44 males, 21 females) who underwent RFA of HCC. Hematologic and biochemical markers were assessed at the pre-RFA period and 1 day, 2-3 days, and 1-2 weeks after RFA. We classified the subjects into two groups: Child-Pugh A $(n=41)$ and Child-Pugh B $(n=24)$. The ablative margin volume (AMV) of each patient was measured. We analyzed the changes in laboratory profiles from the baseline, and investigated whether these laboratory changes were correlated with the AMV and the Child-Pugh classification.

Results: Most of the laboratory values peaked at $2-3$ days after RFA. AMV was significantly correlated with changes in WBC count, hemoglobin level, and serum total bilirubin level (Pearson's correlation coefficient, $0.324-0.453 ; P<0.05$ ). The alanine aminotransferase (ALT) level varied significantly over time $(P=0.023)$.

Conclusions: Most of the measured laboratory markers changed from baseline, peaking at 2-3 days. The ALT level was the only parameter for which there was a significant difference after RFA between Child-Pugh A and B patients: it increased significantly more in the Child-Pugh A patients. (Clin Mol Hepatol 2015;21:71-79)

Keywords: Alanine aminotransferase; Child-Pugh class; Hepatocellular carcinoma; Leukocytosis; Radiofrequency ablation

\section{INTRODUCTION}

Hepatocellular carcinoma (HCC) is the most common primary hepatic malignancy, accounting for $85-90 \%$ of all primary liver cancers, and its incidence is increasing worldwide. 'Radiofrequen- cy ablation (RFA) is a local ablative treatment option that has the potential to be a curative therapy for very early and early BCLC stages of $\mathrm{HCC}^{2}$ RFA is considered a promising option because of its simplicity and effectiveness. ${ }^{3.5}$ One of the main advantages of RFA is the relatively low incidence of treatment-related morbidity

\author{
Abbreviations: \\ AMV, Ablative margin volume; ALT, Alanine aminotransferase; \\ AST, Aspartate aminotransferase; CT, Computed tomography; \\ HCC, Hepatocellular carcinoma; RFA, Radiofrequency ablation; US, \\ Ultrasound; WBC, White blood cell
}

\author{
Corresponding author: Woo Kyoung Jeong \\ Department of Radiology and Center for Imaging Science, Samsung \\ Medical Center, Sungkyunkwan University, School of Medicine, 81 Irwon- \\ ro, Gangnam-gu, Seoul 135-710, Korea \\ Tel: +82-2-3410-1923, Fax: +82-2-3410-0049 \\ E-mail: jeongwk@gmail.com
}


and mortality compared to surgery and transarterial chemoembolization (TACE). ${ }^{6-8}$ Although hepatic resection remains the mainstay of HCC treatment, hepatic resection is not recommended in patients with portal hypertension and hyperbilirubinemia; however, transplantation or local ablative treatments, including RFA and percutaneous ethanol injection, can be used in these patients according to the most recent EASL guideline. ${ }^{2}$ In the setting of adequate technical and clinical experience, RFA is considered effective for the treatment of small-sized HCCs, with overall survival approaching that of surgical resection. ${ }^{9-11}$

However, thermal injury to non-tumor liver tissue is inevitable after RFA, and acute deterioration of functioning hepatocytes can occur. Although the injury is usually mild and self-limited, extensive and multifocal ablation for the treatment of large and multiple HCCs can potentially have a negative impact on the overall reservoir of hepatic function, and may induce clinical symptoms, such as postablation syndrome. ${ }^{12,13}$ To monitor acute liver injury following treatment, biochemical and hematologic profiles are usually obtained both pre- and post-procedure. There have been few studies concerning biochemical and hematologic changes after RFA, ${ }^{14-17}$ and it is well known that ablation volume correlates with acute laboratory changes in non-cirrhotic patients. However, it is unclear how the severity of liver cirrhosis is related to acute hematologic and biochemical changes after RFA. We hypothesized that acute laboratory changes would depend on the severity of the underlying liver disease as well as the tumor ablation volume because the tissue's physical properties, such as electric or heat conductivity, would be different according to the composition of the hepatic parenchyma, such as the amount of hepatic fibrosis. ${ }^{18}$ Thus, the aim of our study was to investigate sequential changes in hematologic and biochemical markers after RFA of HCC and the relationship of these changes to the severity of underlying liver disease when the ablation volume was included as a covariate.

\section{METHODS}

\section{Patients}

The institutional review board of our hospital (Hanyang University Guri Hospital) approved this retrospective study, and the requirement for informed consent was waived. We retrospectively reviewed 78 consecutive patients with 88 HCCs who underwent ultrasound (US)-guided percutaneous RFA at our institution during the period spanning from March 2008 to July 2012. Of these patients, we excluded patients without post-RFA laboratory data $(n=6)$ and patients who underwent additional RFA within 1 week due to insufficient ablative margin $(n=7)$. Ultimately, we included 65 patients (44 men, 21 women; mean age, 61.8 years; age range, 42 to 87 years) with $74 \mathrm{HCCs}$ (Fig. 1). Among them, 58 patients had single HCC, 5 patients had two HCCs, and 2 patients had three HCCs. Sixty-nine nodules in 60 patients were diagnosed as HCC based on typical features in accordance with the EASL guideline. ${ }^{2}$ In three patients, three nodules, which were smaller than $1 \mathrm{~cm}$, showed a typical enhancement pattern on CT or MRI, including arterial enhancement and washout features. The remaining two nodules in two patients, which did not show typical HCC features on imaging, were confirmed by biopsy. The underlying liver diseases of the subjects were as follows: hepatitis B viral infection ( $n=38)$, hepatitis C viral infection $(n=4)$, heavy alcohol use $(n=7)$, and cryptogenic liver cirrhosis $(n=16)$. The severity of the underlying liver diseases was graded using Child-Pugh classification: 41 (63\%) were Child-Pugh A (CPA), 24 (37\%) were Child-Pugh $B(C P B)$, and none were Child-Pugh C.

\section{RFA Procedures}

A radiologist (W.K.J.) with more than five years of experience performed all of the RFA procedures on an inpatient basis. Prior to the RFAs, patients underwent planning US for evaluation of feasibility of RFA. The patients were placed in the supine position and treated under local anesthesia. For pain relief, patients received a continuous intravenous infusion of $50 \mathrm{mg}$ of pethidine hydrochloride (Pethidine; Samsung Pharmaceutical, Seoul, Korea)

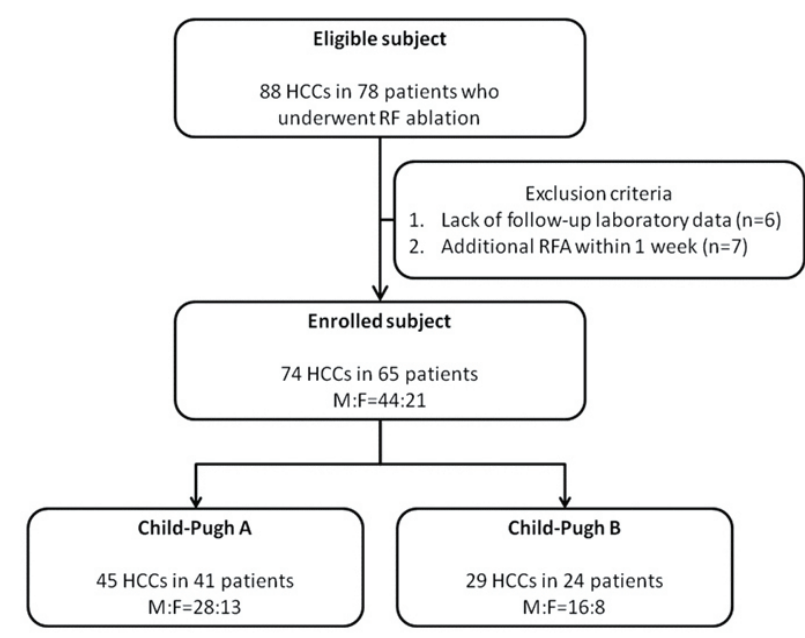

Figure 1. Flowchart of the study population. 
mixed with $50 \mathrm{~mL}$ of $5 \%$ dextrose in water. Two types of RFA electrodes were used. Single $(n=59)$ or cluster $(n=6)$ internally cooled electrodes (Cool-Tip, Covidien, Mansfield, Massachusetts or Well-point, STARmed, Goyang, Korea) were used in this study. After inserting the electrode, the generator power started at 50 W and continuously increased to avoid abruptly increasing intratumoral pressure (popping phenomenon). Most of the HCCs with sizes smaller than $2 \mathrm{~cm}$ underwent single ablation using an electrode that had an exposed length of $3 \mathrm{~cm}$ in the active area of electrode. All ablations aimed to achieve adequate ablation margins, defined as at least $5 \mathrm{~mm}$ from the boundaries of the index tumors. If the ablation margin was not adequate on immediate monitoring after ablation, additional ablation was performed, and these cases were excluded from this investigation as mentioned above. Large HCCs underwent multiple overlapping ablations $(n=21)$ tailored to tumor size and shape.

\section{Laboratory data}

We categorized the hematologic and biochemical tests into four groups, including baseline, 1 day after RFA (1st follow-up), 2-3 days after RFA (2nd follow-up), and 1-2 weeks after RFA (3rd follow-up). Because the number of patients who underwent laboratory tests at 72-168 hours (3-7 days) was too small, we excluded the data from this investigation. If a patient had more than two laboratory results at any time point, the data showing a maximum difference from baseline was selected. Additionally, we investigated changes between the early follow-up laboratory data and the baseline data, i.e. the first change ( $\Delta$ 1st follow-up) and the second change ( $\triangle 2$ nd follow-up).

White blood cell (WBC) count, hemoglobin level, and platelet count were included in the hematologic profiles, and total bilirubin, aspartate aminotransferase (AST), alanine aminotransferase (ALT), and alkaline phosphatase were included in the biochemical profiles.

Table 1. Patient characteristics

\begin{tabular}{|c|c|c|c|}
\hline & Child Pugh A $(n=41)$ & Child Pugh B ( $n=24)$ & $P$-value \\
\hline Age (year) & $60.3 \pm 7.0(48-68)$ & $60.2 \pm 10.2(43-76)$ & 0.777 \\
\hline Gender & & & $>0.999^{*}$ \\
\hline Male & 16 & 28 & \\
\hline Female & 8 & 13 & \\
\hline Number of index tumors & $1.21 \pm 0.51(1-3)$ & $1.10 \pm 0.37(1-3)$ & 0.318 \\
\hline Total tumor volume $(\mathrm{mL})$ & $2.59 \pm 3.14$ & $2.28 \pm 2.62$ & 0.674 \\
\hline Total ablation volume (mL) & $20.20 \pm 10.36$ & $21.33 \pm 11.36$ & 0.693 \\
\hline Ablative margin volume ( $\mathrm{mL})$ & $17.51 \pm 9.38$ & $18.95 \pm 11.25$ & 0.598 \\
\hline Underlying liver disease & & & $0.057^{*}$ \\
\hline Hepatitis B virus & 12 & 26 & \\
\hline Hepatitis C virus & 4 & 0 & \\
\hline Alcoholic liver disease & 2 & 5 & \\
\hline Other causes & 6 & 10 & \\
\hline \multicolumn{4}{|l|}{ Baseline hematologic tests } \\
\hline $\mathrm{WBC}\left(/ \mathrm{mm}^{3}\right)$ & $5136.59 \pm 2184.58$ & $4841.67 \pm 2166.73$ & 0.600 \\
\hline Hemoglobin (g/dL) & $13.26 \pm 1.12$ & $11.09 \pm 2.34$ & $<0.001$ \\
\hline Platelet $\left(/ \mathrm{mm}^{3}\right)$ & $115.12 \mathrm{~K} \pm 56.88 \mathrm{~K}$ & $99.79 K \pm 56.55 K$ & 0.297 \\
\hline \multicolumn{4}{|l|}{ Baseline biochemical tests } \\
\hline Bilirubin (mg/dL) & $0.77 \pm 0.45$ & $1.45 \pm 1.16$ & 0.010 \\
\hline AST (U/L) & $35.49 \pm 17.97$ & $42.38 \pm 15.71$ & 0.124 \\
\hline $\operatorname{ALT}(U / L)$ & $27.90 \pm 21.08$ & $23.58 \pm 16.09$ & 0.392 \\
\hline $\mathrm{ALP}(\mathrm{U} / \mathrm{L})$ & $94.05 \pm 39.88$ & $99.04 \pm 40.53$ & 0.630 \\
\hline
\end{tabular}

WBC, white blood cell; AST, aspartate transaminase; ALT, alanine aminotransferase; ALP, alkaline phosphatase.

${ }^{*} P$-values were calculated from chi-square tests; otherwise independent Student's $t$-test was used. 
Table 2. Hematologic and biochemical laboratory markers (mean \pm SD values)

\begin{tabular}{|c|c|c|}
\hline Time point & Mean & $P$-value \\
\hline \multicolumn{3}{|l|}{$\mathrm{WBC}\left(/ \mathrm{mm}^{3}\right)$} \\
\hline Baseline & $5027 \pm 2166$ & \\
\hline 1 day after RFA & $5572 \pm 2553$ & 0.015 \\
\hline 2-3 days after RFA & $6402 \pm 2362$ & $<0.001$ \\
\hline 7 days after RFA & $4954 \pm 1915$ & 0.611 \\
\hline \multicolumn{3}{|l|}{ Hemoglobin (g/dL) } \\
\hline Baseline & $12.46 \pm 1.97$ & \\
\hline 1 day after RFA & $12.51 \pm 1.84$ & 0.543 \\
\hline 2-3 days after RFA & $12.25 \pm 1.72$ & 0.005 \\
\hline 7 days after RFA & $12.54 \pm 1.66$ & 0.544 \\
\hline \multicolumn{3}{|l|}{ Platelet $\left(/ \mathrm{mm}^{3}\right)$} \\
\hline Baseline & $109,462 \pm 56,808$ & \\
\hline 1 day after RFA & $101,593 \pm 43,515$ & 0.336 \\
\hline 2-3 days after RFA & $92,860 \pm 40,504$ & $<0.001$ \\
\hline 7 days after RFA & $120,356 \pm 60,405$ & 0.270 \\
\hline \multicolumn{3}{|l|}{ Bilirubin (mg/dL) } \\
\hline Baseline & $1.02 \pm 0.85$ & \\
\hline 1 day after RFA & $1.27 \pm 0.91$ & 0.010 \\
\hline 2-3 days after RFA & $1.49 \pm 0.97$ & $<0.001$ \\
\hline 7 days after RFA & $1.11 \pm 0.72$ & 0.242 \\
\hline \multicolumn{3}{|l|}{$\mathrm{AST}(\mathrm{U} / \mathrm{L})$} \\
\hline Baseline & $38.03 \pm 17.37$ & \\
\hline 1 day after RFA & $134.39 \pm 86.85$ & $<0.001$ \\
\hline 2-3 days after RFA & $138.16 \pm 55.41$ & $<0.001$ \\
\hline 7 days after RFA & $40.34 \pm 20.53$ & 0.298 \\
\hline \multicolumn{3}{|l|}{$\mathrm{ALT}(\mathrm{U} / \mathrm{L})$} \\
\hline Baseline & $26.28 \pm 19.34$ & \\
\hline 1 day after RFA & $75.70 \pm 62.51$ & $<0.001$ \\
\hline 2-3 days after RFA & $101.31 \pm 64.23$ & $<0.001$ \\
\hline 7 days after RFA & $31.19 \pm 23.44$ & 0.203 \\
\hline \multicolumn{3}{|l|}{$\mathrm{ALP}(\mathrm{U} / \mathrm{L})$} \\
\hline Baseline & $90.32 \pm 30.67$ & \\
\hline 1 day after RFA & $93.41 \pm 36.85$ & 0.210 \\
\hline 2-3 days after RFA & $106.18 \pm 49.59$ & 0.086 \\
\hline 7 days after RFA & $108.45 \pm 41.07$ & 0.008 \\
\hline
\end{tabular}

$P$-values were calculated using paired $t$-tests for comparison between baseline and each set of follow-up data.

WBC, white blood cell; AST, aspartate transaminase; ALT, alanine aminotransferase; ALP, alkaline phosphatase.

\section{Tumor ablation volume}

Volumes of index tumors and ablation zones were calculated on the pre-procedural and immediate post-procedural CT scans, re- spectively. Two radiologists (S.L and W.K.J.) reviewed the CT images under consensus. The largest diameter of the tumor in three orthogonal planes was measured, and the volume of the tumor and ablation zones were calculated using the following formula: $1 / 6 \times \pi \times a \times b \times c$, where $a, b$, and $c$ were maximum $x, y$, and $z$ axis lengths, respectively. All measurements were made using axial scans and coronal reformatted images acquired during the portal venous phase. The total ablation volume of tumors and ablation zones were calculated by summing the volumes of all treated tumors. The ablative margin volume (AMV) was calculated by subtracting the tumor volume from the ablation zone volume.

\section{Statistical analysis}

Biochemical and hematologic laboratory profiles are shown as mean \pm standard error, and independent Student t-tests and chisquare tests were performed to assess for differences between CPA and CPB groups. Paired Student $t$-tests were used to compare the serial laboratory profiles with the baseline. In addition, Pearson's correlation tests were used to evaluate the association between the ablation volumes and changes in each laboratory profile. An independent Student t-test was also performed for the comparison of mean AMV between CPA and CPB patients. Repeated measure of analysis of covariance (ANCOVA) was used to determine the relationship between changes in laboratory profiles and Child-Pugh classification (CPA vs. CPB) using AMV as a covariate to control for individual variation in ablation volume. Statistical analyses were performed using SPSS for Windows (version 18; SPSS Inc., Chicago, IL, USA). $P$-values $<0.05$ were considered statistically significant.

\section{RESULTS}

\section{Baseline subject characteristics}

Table 1 shows the characteristics and medical records of the 65 study patients. Mean age and standard deviation were 61.8 years and 10.0 years, respectively (range, $42-87$ years). There were 44 male and 21 female patients.

There were no significant differences between the CPA and CPB groups in total tumor volume, total ablation volume, and AMV $(P>0.05)$. The numbers of index tumors per patient were 1.21 in the CPA group and 1.10 in the CPB group (range, 1-3), which was not a significant difference. Although all of the patients with hep- 
Table 3. Coefficients of the correlations between ablative volumes and laboratory values

\begin{tabular}{lcccccccc}
\hline Ablative volume & WBC & Hemoglobin & Platelet & Bilirubin & AST & ALT & ALP \\
\hline Total ablative volume & $0.409^{*}$ & $0.343^{*}$ & 0.151 & $0.451^{*}$ & 0.282 & 0.261 & 0.075 \\
\hline Ablative margin volume & $0.340^{*}$ & $0.324^{*}$ & 0.189 & $0.453^{*}$ & 0.233 & 0.244 & 0.056 \\
\hline
\end{tabular}

WBC, white blood cell; AST, aspartate transaminase; ALT, alanine aminotransferase; ALP, alkaline phosphatase.

*Statistically significant $(P<0.05)$.

Table 4. Differences in mean changes in laboratory values between Child-Pugh A and B patients

\begin{tabular}{|c|c|c|c|c|c|}
\hline & \multicolumn{2}{|c|}{$\Delta$ 1st follow-up } & \multicolumn{2}{|c|}{$\Delta$ 2nd follow-up } & \multirow{2}{*}{$P$-value* } \\
\hline & Child A & Child B & Child A & Child B & \\
\hline WBC $\left(/ \mathrm{mm}^{3}\right)$ & $792 \pm 1732$ & $1927 \pm 2484$ & $1596 \pm 1731$ & $1555 \pm 1828$ & 0.088 \\
\hline Hemoglobin (g/dl) & $-0.19 \pm 0.80$ & $0.33 \pm 0.56$ & $-0.49 \pm 0.71$ & $-0.41 \pm 1.56$ & 0.111 \\
\hline Platelet $\left(/ \mathrm{mm}^{3}\right)$ & $-3,875 \pm 15,845$ & $-13,000 \pm 60,337$ & $-17,167 \pm 21,703$ & $-27,909 \pm 63,341$ & 0.675 \\
\hline Bilirubin (mg/dl) & $0.25 \pm 0.49$ & $0.17 \pm 0.61$ & $0.60 \pm 0.59$ & $0.31 \pm 0.67$ & 0.762 \\
\hline AST (U/L) & $124.4 \pm 96.6$ & $82.1 \pm 71.3$ & $110.9 \pm 56.0$ & $82.1 \pm 45.6$ & 0.098 \\
\hline ALT (U/L) & $71.7 \pm 64.6$ & $28.9 \pm 50.4$ & $98.1 \pm 65.5$ & $39.3 \pm 42.6$ & 0.023 \\
\hline ALP (U/L) & $1.5 \pm 18.5$ & $4.8 \pm 13.1$ & $4.7 \pm 25.7$ & $24.1 \pm 47.8$ & 0.188 \\
\hline
\end{tabular}

$\Delta$ 1st follow-up means the gradient between baseline and 1 day after RFA; $\Delta$ 2nd follow-up means the gradient between baseline and 2-3 day after RFA. " $P$-values were calculated using repeated measurement of covariance (ANCOVA) with marginal ablation volume as a covariate. WBC, white blood cell; AST, aspartate transaminase; ALT, alanine aminotransferase; ALP, alkaline phosphatase.

atitis $C$ infection were included in the CPA group, the difference in underlying liver diseases between groups was not statistically significant.

The mean ablation time was 16.3 minutes (range, 10-48 minutes). There were no serious complications requiring blood transfusion or drainage after the procedure.

\section{Hematologic and biochemical change after RFA}

Table 2 shows mean values of the hematologic and biochemical tests at each time point. Most of biochemical tests, including serum bilirubin, AST, and ALT levels, had changed significantly from baseline at the 1st and 2nd follow-up $(P<0.05)$. The platelet level decreased significantly only on follow-up labs obtained 2-3 days post-RFA. Comparing the changes between each period and baseline laboratory profiles, the maximum differences from baseline values were generally seen 2-3 days after RFA, except for hemoglobin and alkaline phosphatase levels. The hemoglobin level decreased significantly 2-3 days after RFA, but it fluctuated during the immediate post-RFA period. Alkaline phosphatase peaked 1-2 weeks after RFA. The mean values nearly returned to baseline at the 3rd follow-up, with the exception of the alkaline phosphatase level.

\section{Ablation volume and analysis after adjusting for volume}

Ablative volumes including AMV had significantly positive correlations with changes in the WBC count, hemoglobin level, and serum total bilirubin level (Table 3). AST and ALT did not significantly correlate with ablation volumes. Mean AMV in CPA patients was not significantly different from that in CPB patients ( $P=0.598)$.

Comparing $\Delta$ 1st and 2nd follow-up data using repeated measure of covariance (ANCOVA) with AMV as a covariate (Table 4), the change in serum ALT level was only significantly different between CPA and CPB $(P=0.023)$. The change from baseline at 1 day after RFA was about $73 \mathrm{U} / \mathrm{L}$ in the CPA group and $29 \mathrm{U} / \mathrm{L}$ in the CPB group, and the difference between baseline and 2-3 days was about $100 \mathrm{U} / \mathrm{L}$ in the CPA group and $39 \mathrm{U} / \mathrm{L}$ in the CPB group (Fig. 2 and 3).

\section{DISCUSSION}

This study evaluated acute changes in hematologic and biochemical data in the first week following RFA treatment in patients with chronic liver diseases and investigated the relationship between laboratory changes and the severity of underlying liver 
A

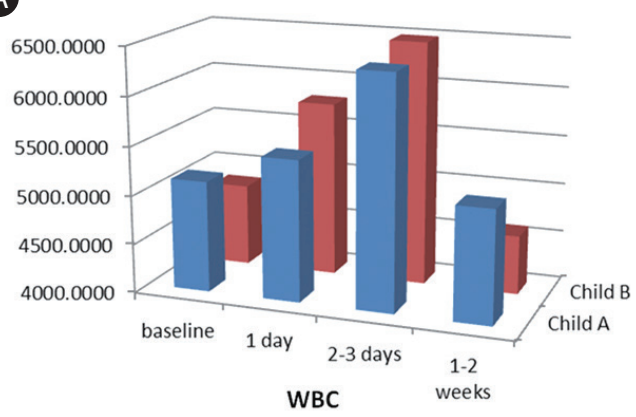

C

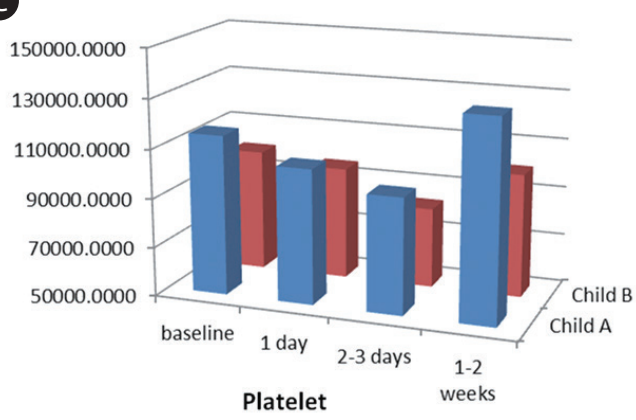

E

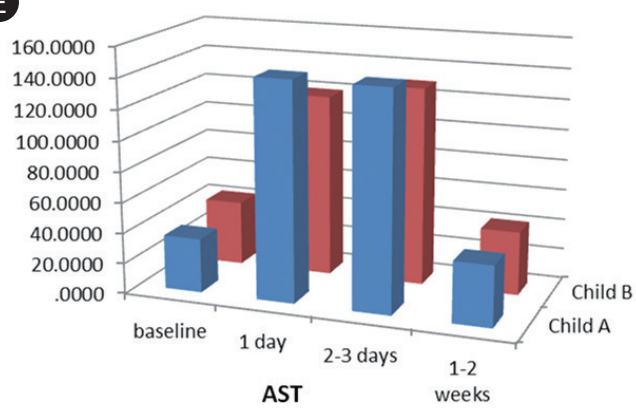

G

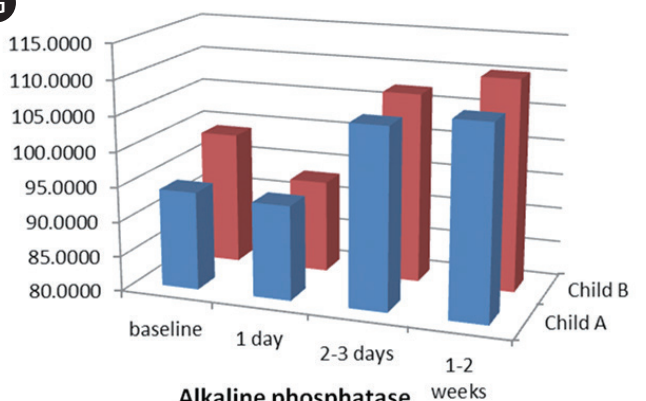

diseases, as assessed by Child-Pugh classification. We demonstrated that most of the laboratory changes after RFA peaked 2-3 days and returned to baseline about 1 week post-RFA. In our results, serum AST and ALT levels were prominently elevated, and the AST level increased earlier and reached a higher peak than the ALT value. Generally, serum AST and ALT levels are elevated when

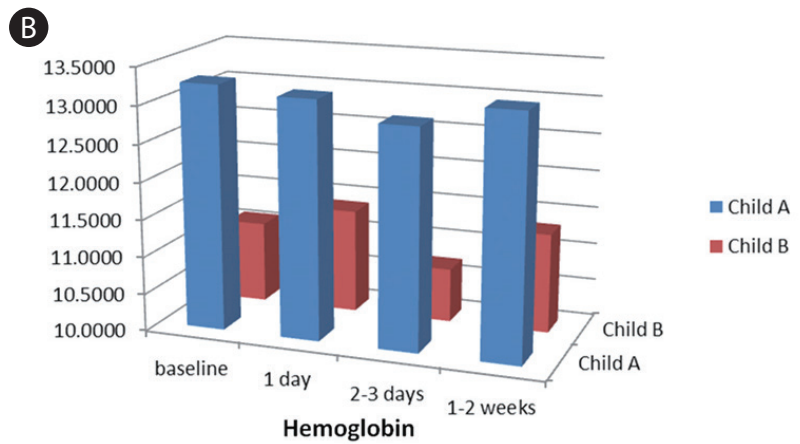

(D)

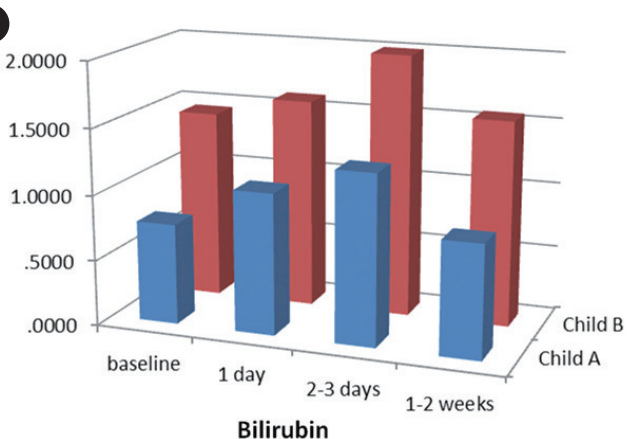

Child A

- Child A

Child B

- Child A

- Child B

F

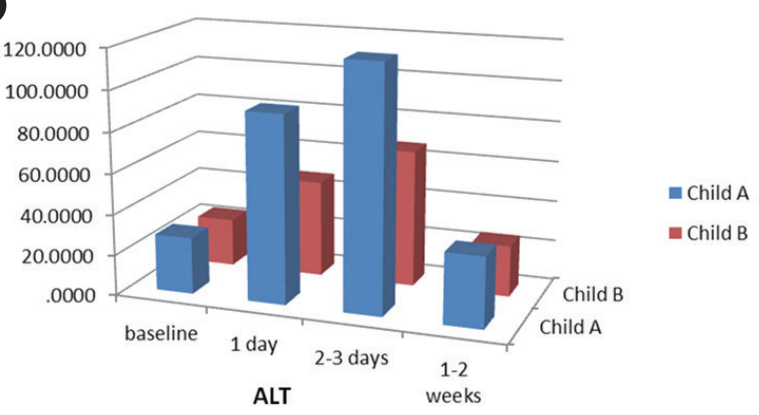

- Child A

a Child B

Figure 2. Comparison of serial hematologic and biochemical values (AG) according to Child-Pugh classification. The baseline hemoglobin (B) and serum bilirubin (D) levels differed significantly between the classified groups. WBC, White blood cell; AST, Aspartate aminotransferase; ALT, Alanine aminotransferase.

hepatocytes are injured and become necrotic by various causes, such as hepatotoxic drugs, virus-related necroinflammation, or physical injury including hyperthermia by RFA. ${ }^{16,17}$ AST is widely distributed not only in the liver but also in the heart and skeletal muscle, while ALT is known to be a more specific test for detecting acute liver disease. ${ }^{19-21}$ In particular, AST activity is about three 
A

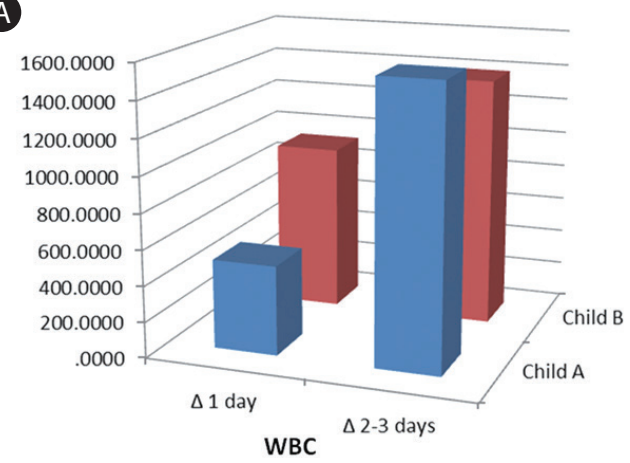

C

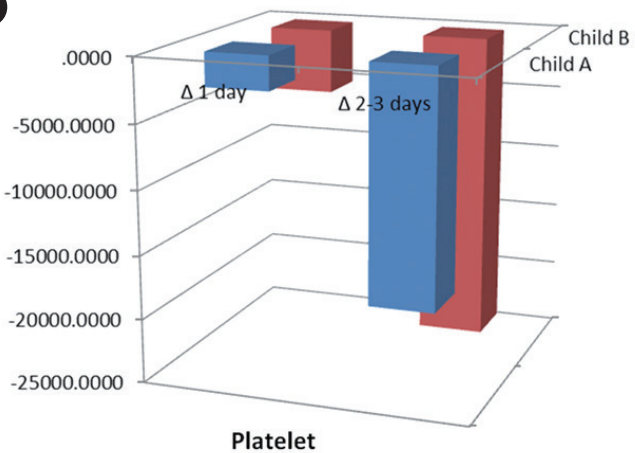

E

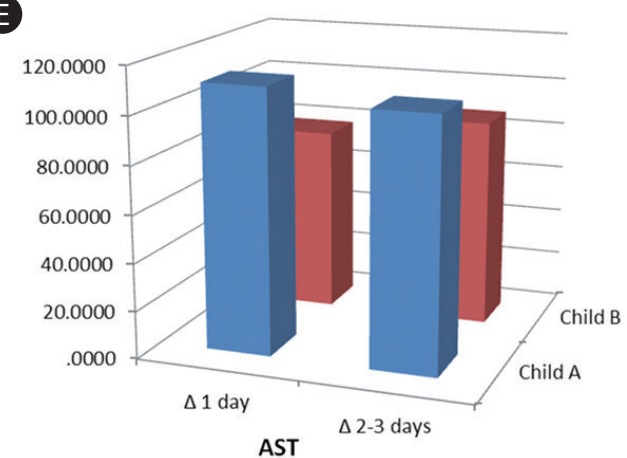

G

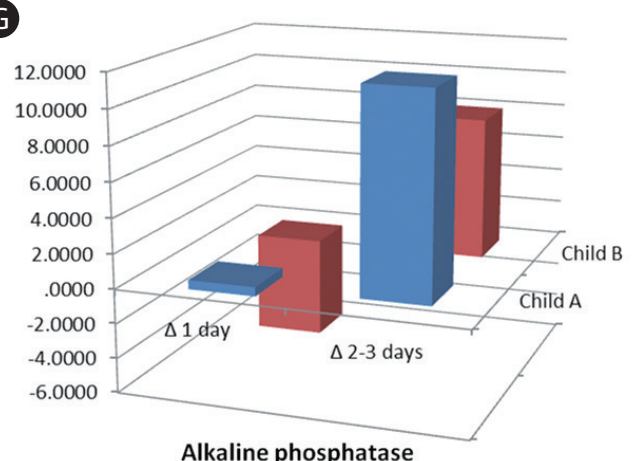

times that of ALT in hepatocytes. ${ }^{19}$ Peak AST activity typically precedes ALT in the setting of hepatic injury and declines rapidly because the half-life of AST is shorter than that of ALT. ${ }^{21}$

In our study, the serum ALT level increased slowly and the serum a Child A

a Child B
B

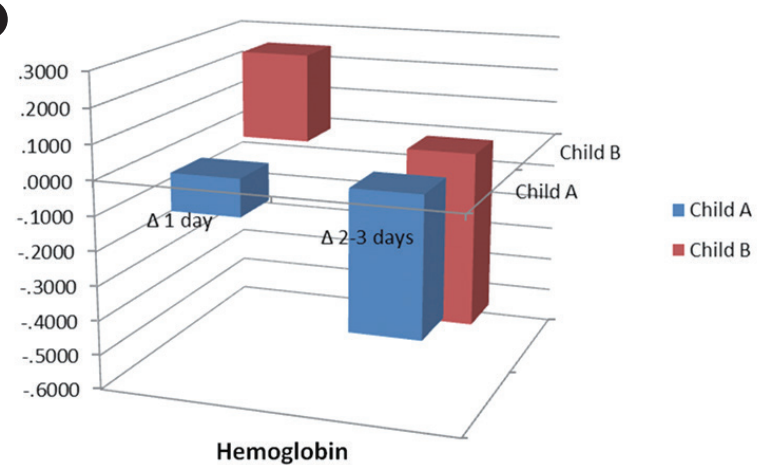

(D)

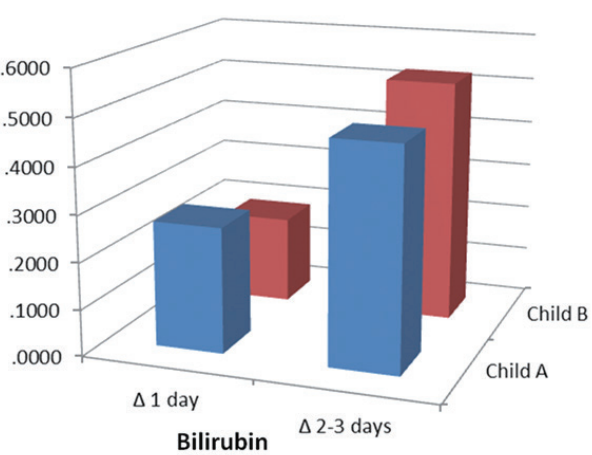

F

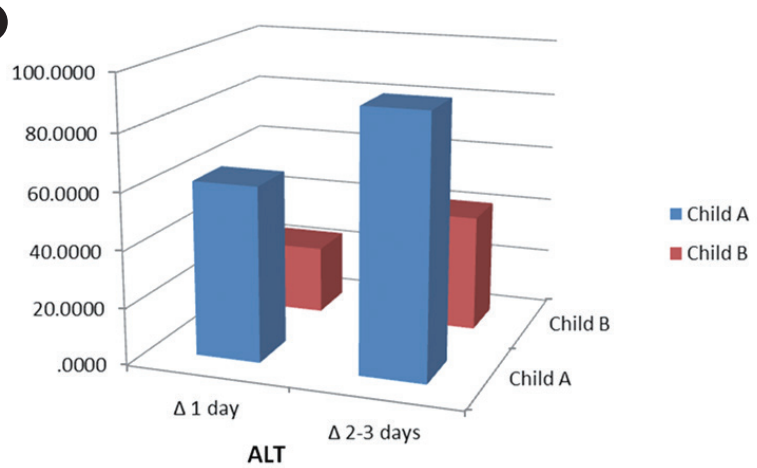


an oven effect. The cirrhotic liver parenchyma around the tumor may affect heat transmission. Lower thermal conductivity of the background cirrhotic tissue leads to delayed heat transmission from the central tumor portion to the surrounding non-tumor liver tissue. $^{22,23}$ Liu et al. have demonstrated that increasing thermal conductivity in the outer surrounding tissue permitted greater heat transmission in their ex-vivo study. ${ }^{22}$ Moreover, an excellent correlation was recently reported between histological subclassification of cirrhosis and Child-Pugh classification. ${ }^{24}$ Therefore, if the patients in the CPB group had more severely cirrhotic liver parenchyma than those in the CPA group, this would explain the steeper ALT elevation in the CPA patients than in the CPB patients.

The serum bilirubin level was observed to be elevated immediately after RFA, as has been noted in several previous studies, ${ }^{25,26}$ but serum alkaline phosphatase increased slowly and its peak was observed 1 week after ablation. These results are supported by a previous study and are potentially consistent with minor injury of the small bile duct and cholestasis resulting from hepatocyte necrosis, although there was no gross evidence of biliary injury on follow-up CT. ${ }^{17}$

In the analysis of the relationship of the ablative margin volume to laboratory studies, we found that WBC count, hemoglobin, and bilirubin level were significantly correlated with ablative margin volume. Mild leukocytosis after RFA was reported and was clinically inconsequential and self-limited. ${ }^{17}$ It might be attributed to inflammatory phenomena associated with tissue repair. ${ }^{27} \mathrm{~A}$ postprocedural decrease in hemoglobin level was also noted, but was subclinical and may have been caused by blood loss during RFA and hemodilution due to fluid treatment. It is reasonable to expect that these hematologic changes would correlate with ablation volume but not with the severity of the underlying liver disease. Some of the baseline hematologic tests were lower in the CPB group, but the changes in the hematologic parameters, including hemoglobin and platelet count, after RFA were not different between the CPA and CPB groups after adjusting for AMV. Lastly, bilirubin is an important indicator of developing hepatic failure after treatment, such as TACE or hepatic resection. ${ }^{7,28}$ It was also elevated after RFA in this study and relatively well correlated with AMV. However, the change in bilirubin was not significantly different between the CPA and CPB groups, although the baseline levels were significantly different. Bilirubin normalized 1-2 weeks after RFA, perhaps because synthetic dysfunction after RFA might be less than after TACE or hepatic resection.

There was a significant decrease in platelet count in our study that returned to baseline 1-2 weeks after RFA. During the first 2-3 days, the mean decrease was about $25,000 / \mathrm{mm}^{3}$ in the CPB group. Because a platelet count below $50,000 / \mathrm{mm}^{3}$ significantly increases the risk of spontaneous bleeding, including GI bleeding $_{1}{ }^{16}$ and the change in the CPB group was larger than in the CPA group, although not statistically significant, we should be careful to assess for internal bleeding during the immediate follow-up period after patients with high Child-Pugh scores undergo RFA.

This study had several limitations. First, the study was retrospective; we could not fix the times for serial laboratory tests, but rather obtained data at baseline, 1 day, 2-3 days, and 1-2 weeks after RFA. Second, the relationship of tumor ablation zone with the central bile duct was not considered in this study. There is potential for the bile duct to be destroyed by thermal injury and it results in bilirubin elevation. In this study, serious complications involving bile duct injury were not observed on follow-up CT after RFA. Finally, we could not consider the "heat sink effect." It has been demonstrated that large blood vessels adjacent to HCCs convect heat from the ablation zone to tissue. ${ }^{29,30}$ Therefore, this could confound the relationship between cirrhosis and laboratory changes.

In conclusion, most of the measured hematologic and biochemical markers related to liver function peaked 2-3 days after RFA, and the change in serum ALT level was significantly higher in CPA patients than in patients with severe hepatic cirrhosis.

\section{Conflicts of Interest}

The authors have no conflicts to disclose.

\section{REFERENCES}

1. El-Serag HB, Mason AC. Rising incidence of hepatocellular carcinoma in the United States. N Engl J Med 1999;340:745-750.

2. EASL-EORTC clinical practice guidelines: management of hepatocellular carcinoma. J Hepatol 2012;56:908-943.

3. Wood TF, Rose DM, Chung M, Allegra DP, Foshag LJ, Bilchik AJ. Radiofrequency ablation of 231 unresectable hepatic tumors: indications, limitations, and complications. Ann Surg Oncol 2000;7:593-600.

4. Lencioni R, Crocetti L, Cioni D, Della Pina C, Bartolozzi C. Percutaneous radiofrequency ablation of hepatic colorectal metastases: technique, indications, results, and new promises. Invest Radiol 2004;39:689-697.

5. Howard JH, Tzeng CW, Smith JK, Eckhoff DE, Bynon JS, Wang T, et al. Radiofrequency ablation for unresectable tumors of the liver. Am Surg 2008;74:594-600; discussion 600-601.

6. Fan ST, Lo CM, Liu CL, Lam CM, Yuen WK, Yeung C, et al. Hepatectomy for hepatocellular carcinoma: toward zero hospital deaths. Ann Surg 1999;229:322-330. 
7. Hsin IF, Hsu CY, Huang HC, Huang $Y H$, Lin $H C$, Lee RC, et al. Liver failure after transarterial chemoembolization for patients with hepatocellular carcinoma and ascites: incidence, risk factors, and prognostic prediction. J Clin Gastroenterol 2011;45:556-562.

8. Garwood ER, Fidelman N, Hoch SE, Kerlan RK Jr, Yao FY. Morbidity and mortality following transarterial liver chemoembolization in patients with hepatocellular carcinoma and synthetic hepatic dysfunction. Liver Transpl 2013;19:164-173.

9. Chen MS, Li JQ, Zheng Y, Guo RP, Liang HH, Zhang YQ, et al. A prospective randomized trial comparing percutaneous local ablative therapy and partial hepatectomy for small hepatocellular carcinoma. Ann Surg 2006;243:321-328.

10. Nicoli N, Casaril A, Marchiori L, Mangiante G, Marini G, Colombari R, et al. Intraoperative and percutaneous radiofrequency thermal ablation in the treatment of hepatocellular carcinoma. Chir Ital 2000;52:29-40.

11. Buscarini L, Buscarini E, Di Stasi M, Vallisa D, Quaretti P, Rocca A. Percutaneous radiofrequency ablation of small hepatocellular carcinoma: long-term results. Eur Radiol 2001;11:914-921.

12. Dodd GD, 3rd, Napier D, Schoolfield JD, Hubbard L. Percutaneous radiofrequency ablation of hepatic tumors: postablation syndrome. AJR Am J Roentgenol 2005;185:51-57.

13. Seror O, N'Kontchou G, Ibraheem M, Ajavon Y, Barrucand C, Ganne $\mathrm{N}$, et al. Large $(>0 \mathrm{r}=5.0-\mathrm{cm}) \mathrm{HCC}$ : multipolar RF ablation with three internally cooled bipolar electrodes--initial experience in 26 patients. Radiology 2008;248:288-296.

14. Koike Y, Yoshida H, Shiina S, Teratani T, Obi S, Sato S, et al. Changes in hepatic functional reserve after percutaneous tumor ablation for hepatocellular carcinoma: long-term follow up for 227 consecutive patients with a single lesion. Hepatol Int 2007;1:295-301.

15. Kuroda H, Kasai K, Kakisaka K, Yasumi Y, Kataoka K, Ushio A, et al. Changes in liver function parameters after percutaneous radiofrequency ablation therapy in patients with hepatocellular carcinoma. Hepatol Res 2010;40:550-554.

16. Lee HS, Park SY, Kim SK, Kweon YO, Tak WY, Cho CM, et al. Thrombocytopenia represents a risk for deterioration of liver function after radiofrequency ablation in patients with hepatocellular carcinoma. Clin Mol Hepatol 2012;18:302-308.

17. Cizginer $S$, Tatli $S$, Hurwitz $S$, Tuncali $K$, vanSonnenberg $E$, Silverman SG. Biochemical and hematologic changes after percutaneous radiofrequency ablation of liver tumors: experience in 83 procedures. J Vasc Interv Radiol 2011;22:471-478.

18. Rhim H, Goldberg SN, Dodd GD 3rd, Solbiati L, Lim HK, Tonolini M, et al. Essential techniques for successful radio-frequency thermal ablation of malignant hepatic tumors. Radiographics 2001;21 Spec No:S17-35; discussion S36-39.

19. Wolf PL. Biochemical diagnosis of liver disease. Indian J Clin Biochem 1999;14:59-90.

20. Rozen P, Korn RJ, Zimmerman HJ. Computer analysis of liver function tests and their interrelationships in 347 cases of viral hepatitis. Isr J Med Sci 1970;6:67-79.

21. Dufour DR, Lott JA, Nolte FS, Gretch DR, Koff RS, Seeff LB. Diagnosis and monitoring of hepatic injury. II. Recommendations for use of laboratory tests in screening, diagnosis, and monitoring. Clin Chem 2000;46:2050-2068.

22. Liu Z, Ahmed M, Weinstein Y, Yi M, Mahajan RL, Goldberg SN. Characterization of the RF ablation-induced 'oven effect': the importance of background tissue thermal conductivity on tissue heating. Int J Hyperthermia 2006;22:327-342.

23. Livraghi T, Goldberg SN, Lazzaroni S, Meloni F, Solbiati L, Gazelle GS. Small hepatocellular carcinoma: treatment with radio-frequency ablation versus ethanol injection. Radiology 1999;210:655-661.

24. Kim MY, Cho MY, Baik SK, Park HJ, Jeon HK, Im CK, et al. Histological subclassification of cirrhosis using the Laennec fibrosis scoring system correlates with clinical stage and grade of portal hypertension. J Hepatol 2011;55:1004-1009.

25. Curley SA, Izzo F, Delrio P, Ellis LM, Granchi J, Vallone P, et al. Radiofrequency ablation of unresectable primary and metastatic hepatic malignancies: results in 123 patients. Ann Surg 1999;230:1-8.

26. Curley SA, Izzo F, Ellis LM, Nicolas Vauthey J, Vallone P. Radiofrequency ablation of hepatocellular cancer in 110 patients with cirrhosis. Ann Surg 2000;232:381-391.

27. Livraghi T, Goldberg SN, Lazzaroni S, Meloni F, lerace T, Solbiati L, et al. Hepatocellular carcinoma: radio-frequency ablation of medium and large lesions. Radiology 2000;214:761-768.

28. Jarnagin WR, Gonen M, Fong Y, DeMatteo RP, Ben-Porat L, Little S, et al. Improvement in perioperative outcome after hepatic resection: analysis of 1,803 consecutive cases over the past decade. Ann Surg 2002;236:397-406; discussion 406-397.

29. Goldberg SN, Hahn PF, Tanabe KK, Mueller PR, Schima W, Athanasoulis $C A$, et al. Percutaneous radiofrequency tissue ablation: does perfusionmediated tissue cooling limit coagulation necrosis? J Vasc Interv Radiol 1998:9:101-111.

30. Goldberg SN, Hahn PF, Halpern EF, Fogle RM, Gazelle GS. Radiofrequency tissue ablation: effect of pharmacologic modulation of blood flow on coagulation diameter. Radiology 1998;209:761-767. 